\title{
EVIDENCIAS DE COMPROMISO CEREBRAL EN EL ESTADIO CRÓNICO DE LA ENFERMEDAD DE CHAGAS OBTENIDAS POR MEDIO DEL POTENCIAL P 300 Y DE ELECTROENCEFALOGRAFÍA CUANTIFICADA
}

\author{
JULIO OSCARPROST*, HORACIO ROMERO VILLANUEVA **, \\ ANA MARIA MORIKONE***, GUSTAVO POLO****, ANA MARIA BOSCH***
}

RESUMEN - Es reconocido el compromiso del sistema nervioso periférico en la etapa crónica de la enfermedad de Chagas, incorporándose últimamente evidencias tomográficas y neuropsicológicas de compromiso cerebral. Con el objetivo de evaluar dicho compromiso por medio del potencial P 300 y la electroencefalografía cuantificada (EEGc) se estudiaron 35 pacientes ( 26 a 55 años), comparados con un grupo control de similar número y edad ( 29 a 55 años). Se observó: latencia de la onda P 3 mayor en el grupo en estudio $(331,24 \pm 24,02$ contra 318,86 $\pm 23,18)(\mathrm{p}=0,01716)$. El EEGc mostró diferencias en la potencia relativa de la banda Beta 1, menor en los pacientes ( $\mathrm{p}=1,62834 \mathrm{E}-5)$ y en la frecuencia dominante, $1 \mathrm{~Hz}$ menor en los chagasicos ( $\mathrm{p}=0,01077)$. El análisis multivariado discrimina tres subpoblaciones: una normal, una de enfermos con incremento alfa y otra de enfermos con decremento alfa e incremento delta y theta $(\mathrm{p}=0,00004)$. La proporción de resultados patológicos fue del $20 \%$ en los EEGc y el 11,43\% en los potenciales cognitivos. No se correlacionó el compromiso neurológico y el cardíaco. Se concluye que existen francos indicadores electrofisiológicos de compromiso cerebral en el estadio crónico de la enfermedad de Chagas, hallazgo que refuerza a los obtenidos por otros métodos.

PALABRAS-CLAVE: enfermedad de Chagas, sistema nervioso central, potencial p300, electroencefalografía cuantificada (EEGc), mapeo cerebral.

P300 event related potentials and qEEG evidenced a cerebral dysfunction in human chronic Chagas disease

ABSTRACT - It is already known the involvement of the peripheral nervous system in chronical stages of Chagas disease. Tomographic and neuropsychological evidence of brain compromise has been included recently. In order to evaluate the neurophysiological counterpart of cerebral involvement, we studied P300 evoked potential and quantified EEG (qEEG) of 35 patients (26-55 years), and compared to an equal number of control subjects (29-55 years). We have found increased P300 latency compared to the control group (331.24 \pm 24.02 vs 318.86 $\pm 23.18)(\mathrm{p}=0.01716)$. $\mathrm{qEEG}$ showed lower relative Beta 1 power in the patients group $(\mathrm{p}=1.6 \mathrm{E}-5)$, and the principal frequency $1 \mathrm{~Hz}$ slower in the same group $(\mathrm{p}=0.01077)$. Multivariate analysis showed three subpopulations: a normal one, pathological one with higher Alpha power and pathological with Alpha decrement and Delta-Theta increment. Pathological findings represented $20 \%$ for the qEEG and $11.43 \%$ for cognitive potentials. Cardiac and neurologic involvement were not correlated. We conclude that there is clear electrophysiological evidence of cerebral involvement in chronic Chagas disease, thus reinforcing findings obtained by other methods.

KEY WORDS: Chagas disease, central nervous system, event related potential P 300, quantitative electroencephalography (qEEG), brain mapping.

Hospital Bernardino Rivadavia, Buenos Aires, Argentina (Las Heras 2670, 1425 Buenos Aires, Argentina hrsneu@intramed.net.ar): *Médico Jefe Sección de Neurofisiología - Servicio de Neurología; **Médico Jefe Servicio de Cardiología ***Médica Servicio de Cardiología; ****Médico Servicio de Neurología. Aceite: 8dezembro-1999.

Dr. Julio Oscar Prost - Alvarez Jonte 243 PB2 - 1416 Buenos Aires - Argentina. 
La enfermedad de Chagas, en la mayoría de los casos, cursa en forma inaparente, pasando a la etapa crónica, que tras algunos años provoca compromiso miocárdico (prevalente en Argentina) o digestivo, con megaesófago y megacolon (mas frecuente en Brasil). Diversos autores han hecho incapié en el compromiso del sistema nervioso en esta etapa crónica. El mismo, lento y solapado, debe diferenciarse de complicaciones en inmunosuprimidos, que se comportan como una infección aguda o por presencia de masas tumorales (chagomas) ${ }^{1-6}$ El mas estudiado ha sido el del sistema nervioso periférico, detectado en el humano y en modelos animales ${ }^{7,8}$. El mismo consiste fundamentalmente en la agresión de la motoneurona alfa del cuerno anterior medular.

Desde hace años existen informes clínicos sobre sintomatología crónica o secuelar (por ejemplo cefaleas, oligofrenias, convulsiones, paraparesias, hemiparesias $)^{9-12} \mathrm{o}$ alteraciones electroencefalográficas inespecíficas ${ }^{13}$, sin que se haya establecido un cuadro neurológico central claro e inequívoco. En fecha mucho mas reciente se detectaron, por medio de potenciales evocados, alteraciones en la conducción a distintos niveles del sistema nervioso central ${ }^{14}$. Se ha reportado igualmente compromiso de la función cortical por medio de pruebas neuropsicológicas, siendo éste el terreno aún menos explorado ${ }^{15-17}$.

La electroneurofisiología dispone en este momento de herramientas idóneas para evaluar la función cortical. Se han acumulado suficientes conocimientos sobre el electroencefalograma cuantificado - mapeo cerebral y los potenciales evocados cognitivos (P 300), para esta evaluación. El EEG cuantificado (EEGc) permite investigar todos los eventos estacionarios (no paroxismales) del EEG, siendo idónea por lo tanto para evaluar el ritmo de base del mismo y, a partir de este extraer conclusiones sobre el funcionamiento cerebral en diversas situaciones clínicas. El potencial P 300 es un potencial endógeno, generado a partir de una tarea de discriminación entre un estímulo "target" y otro "no target". La latencia de la onda P3 o P300 de este potencial es un indicador de la velocidad de procesamiento cortical. La misma se retarda progresivamente con la edad y muestra una franca prolongación en los cuadros de deterioro cognoscitivo ${ }^{18,20}$.

La presente investigación se planificó con el objetivo de determinar la existencia, en estudios electrofisiológicos, de signos de compromiso de la función cortical cerebral en pacientes portadores de enfermedad de Chagas crónica.

\section{MÉTODO}

\section{Pacientes}

Los pacientes fueron ingresados por el consultorio externo de cardiología, seleccionados según los criterios del Consejo Argentino de Miocardiopatía de Chagas e Infecciosas, de la Sociedad Argentina de Cardiología. El diagnóstico se basó en la positividad de al menos dos de las tres reacciones serológicas efectuadas (inmunoensayo enzimático, hemoaglutinación indirecta cuantitativa e inmunofluorescencia indirecta cuantitativa). Se efectuó examen clínico general, cardiológico y neurológico y una cuidadosa anamnesis y los exámenes de rutina para descartar patologías asociadas que pudieran interferir con los resultados. Fueron excluidos del estudio los sujetos con antecedentes de enfermedades psiquiátricas, vasculares, metabólicas, o historia previa de afección del sistema nervioso central y aquellos con antecedentes de ingesta de alcohol, o con exposición a tóxicos. Ninguno de los pacientes recibía, al momento del estudio, medicación en forma habitual. Desde el punto de vista cardiológico, adecuado a las necesidades de cada paciente, se efectuaron: prueba ergométrica graduada, ecocardiograma, ecodoppler, "holter" de 24 hs y ecografía hepática.

A partir de estos resultados los pacientes fueron catalogados de acuerdo a la Clasificación de la Miocardiopatía Chagásica Crónica; Grupo I: paciente con serología positiva, asintomático, ECG normal y ausencia de alteración cardiovascular en la RX de tórax; Grupo II: pacientes con serología positiva, sin insuficiencia cardíaca, con ECG patológico (subgrupo A: alteraciones en la conducción ventricular; subgrupo B: arritmias ventriculares; y subgrupo C: características de los subgrupos A y B con Rx de tórax con relación cardiotoráxica menor a 0,55); Grupo III: serología positiva, insuficiencia cardíaca congestiva, ECG patológico y cardiomegalia mayor a 0,55 por radiología.

Los pacientes se distribuyeron de la siguiente manera - I: 77,14\%; IIA: 14,29\%; IIB: 2,86\%; IIC: 2,86\%; y III: 2,86 \%. Se efectuó un potencial cognitivo P300, por medio de un equipo ATI Nautilus PE. Se estimuló por 
medio de un paradigma "odd-ball", por cuenta mental, tonal ("burst", $5 \mathrm{~ms}$ de rampa, $20 \mathrm{~ms}$ de "plateau"; frecuente $=1000 \mathrm{~Hz}$, raro $=2000 \mathrm{~Hz}$, con una relación 5:1), recogido en la derivación Cz-M1, banda pasante de 0,5 a $125 \mathrm{~Hz}$ y se analizaron $\operatorname{los} 800 \mathrm{~ms}$ posteriores al estímulo.

El EEGc se obtuvo por medio de un equipo ATI Nautilus de poligrafía, con 16 electrodos en cuero cabelludo, según el sistema 10-20, con referencia biauricular, con el paciente en reposo vigil, ojos cerrados, en decúbito dorsal. Se analizaron al menos 15 épocas libres de artefacto de 4 segundos cada una. Se efectuó una transformación rápida de Fourier y el promedio de dichas muestras. Se estudiaron el espectro de potencias relativas por canal (potencia total de la banda en relación a la potencia total del espectro de $0,5 \mathrm{a} 32 \mathrm{~Hz}$ ), dividido en las bandas electroencefalográficas: delta $(0,5 \mathrm{a} 3,75 \mathrm{~Hz})$, theta $(4,0$ a $7,75 \mathrm{~Hz})$, alfa $(8,0 \mathrm{a} 12,75 \mathrm{~Hz})$, beta1 $(13,0$ a $18,0 \mathrm{~Hz})$ y beta2 $(18,5 \mathrm{a} 32,0 \mathrm{~Hz})$ y el promedio total de las mismas para todos los canales y el mapeo topográfico para el análisis de asimetrías.

Se utilizaron potencias relativas, en lugar de sus valores absolutos, ya que el uso de las mismas disminuye la varianza, con mejor definición de los límites de la normalidad y facilita la comparación de resultados obtenidos con distintos equipos. ${ }^{21}$

Variables exploradas

P 300: latencia al acmé de la onda P3 “target” (en ms) y amplitud pico a pico (N2-P3) de la misma (en $\mu$ s). Mapeo cerebral: potencia relativa de las bandas delta, theta, alfa, beta1 y beta2, frecuencia dominante (en $\mathrm{Hz}$ y, como variable secundaria la relación alfa/theta (cociente).

Cardiopatía: presencia o ausencia de la misma (variable dicotómica).

\section{Estadísticas}

Comparación univariada: se utilizó la prueba U de Mann-Whitney (o suma de rangos de Wilcoxon). Análisis multivariado: se aplicaron el análisis de componentes principales y el análisis discriminante. Se utilizó el programa Statgraphics versión 3.

\section{Proporción de estudios patológicos}

A fin de evaluar el impacto clínico de los hallazgos se evaluó la proporción de pacientes con resultados patológicos en cualquiera de los dos estudios. En el potencial P 300 se consideró patológica la prolongación de la latencia mas alla del tercer desvío standard para la edad del sujeto, en relación a los valores normales para el laboratorio. ${ }^{22}$ En el mapeo cerebral se consideraron como patológicas aquellas alteraciones que se aceptan como indicadoras inequívocas de deterioro de la función bioeléctrica cortical, la inversión de la relación alfa/theta y el corrimiento de la frecuencia dominante a valores menores a $8 \mathrm{~Hz}$.

Población

Se estudiaron 35 pacientes ( 25 de sexo femenino), con edades entre 26 y los 55 años (media 44,02). El grupo control se encontraba formado por 35 sujetos ( 24 mujeres), con edades entre 29 y 55 años (media 43,58), con similares niveles socioculturales y años de escolaridad que el grupo en estudio.

\section{Reparos éticos}

Los pacientes fueron informados de las características de la investigación, dando su consentimiento posterior a la misma; las prácticas efectuadas carecen de riesgos y son no invasivas; habiendo sido aprobada su realización por el Departamento de Docencia e Investigación.

\section{RESULTADOS}

\section{Análisis univariado}

El potencial P 300 mostró diferencias en la latencia de la onda P3, (mayor en el grupo de pacientes) pero no en la amplitud de la misma (Tabla 1).

En el EEGc (Tabla 2) se observa menor potencia relativa en la banda beta, especialmente en beta 1 y menor frecuencia dominante en el grupo de pacientes.

Se evaluó si existían diferencias en el análisis univariado entre sujetos con o sin compromiso cardíaco, sin encontrar diferencias (Tabla 3). 
Tabla 1. Media y dispersión de las variables del potencial P300, asi como su nivel de significación en la comparación univariada

\begin{tabular}{|c|c|c|c|c|c|}
\hline \multirow{2}{*}{ Variable } & \multicolumn{2}{|c|}{ Pacientes } & \multicolumn{2}{|c|}{ Controles } & \multirow{2}{*}{$\mathrm{p}$} \\
\hline & media & d.s. & media & d.s & \\
\hline Latencia P 3 & 331,24 & 24,02 & 318,86 & 23,18 & 0,017167 \\
\hline Amplitud P 3 & 8,17 & 17,07 & 8,93 & 18,45 & 0,497628 \\
\hline
\end{tabular}

Tabla 2. Media y dispersión de las variables del mapeo cerebral, así como su nivel de significación en la comparación univariada

\begin{tabular}{|c|c|c|c|c|c|}
\hline \multirow{2}{*}{ Variable } & \multicolumn{2}{|c|}{ Pacientes } & \multicolumn{2}{|c|}{ Controles } & \multirow{2}{*}{$\mathrm{p}$} \\
\hline & media & d.s. & media & d.s & \\
\hline Potencia relativa delta & 18,7 & 9,5 & 17,3 & 6,5 & 0,953057 \\
\hline Potencia relativa theta & 16,0 & 6,93 & 14,08 & 4,9 & 0,426474 \\
\hline Potencia relativa alfa & 41,03 & 19,15 & 39,28 & 9,67 & 0,759847 \\
\hline Potencia relativa beta 1 & 6,8 & 3,09 & 11,71 & 5,11 & $1,62834 \mathrm{E}-5$ \\
\hline Potencia relativa beta 2 & 8,46 & 5,31 & 11,6 & 6,38 & 0,0284846 \\
\hline Relación alfa/theta & 3,74 & 3,34 & 3,26 & 1,87 & 0,680923 \\
\hline Frecuencia dominante & 8,32 & 1,93 & 9,61 & 1,17 & 0,0107702 \\
\hline
\end{tabular}

Tabla 3. Variables exploradas en el grupo de pacientes chagásicos, sin y con cardiopatía.

\begin{tabular}{lccc}
\hline Variable & Sin cardiopatía & Con cardiopatía & Significación \\
\hline Potencia relativa delta & $18,62 \pm 8,75$ & $19,12 \pm 12,68$ & 0,400812 \\
Potencia relativa theta & $14,81 \pm 6,48$ & $20,12 \pm 7,69$ & 0,674421 \\
Potencia relativa alfa & $42,03 \pm 19,16$ & $37,62 \pm 21,29$ & 0,674421 \\
Potencia relativa beta 1 & $6,77 \pm 3,09$ & $6,87 \pm 3,48$ & 0,529365 \\
Potencia relativa beta 2 & $8,88 \pm 5,87$ & $7,00 \pm 3,16$ & 0,107327 \\
Relación alfa/theta & $3,95 \pm 3,24$ & $3,05 \pm 4,00$ & 0,624061 \\
Frecuencia dominante & $8,27 \pm 2,06$ & $8,50 \pm 1,64$ & 0,352541 \\
Latencia P 3 & $333,27 \pm 26,50$ & $323,40 \pm 11,71$ & 0,554110 \\
\hline
\end{tabular}

Valores expresados en media y d.s.

\section{Análisis multivariado}

El análisis de componentes principales muestra una distribución en tres grupos.

Uno de los mismos se constituye especialmente por los sujetos control y los otros dos especialmente por los pacientes en estudio (Figura 1). La diferencia entre los mismos se da fundamentalmente por el peso de las potencias relativas de las bandas rápidas (mayor en el grupo de normales). 


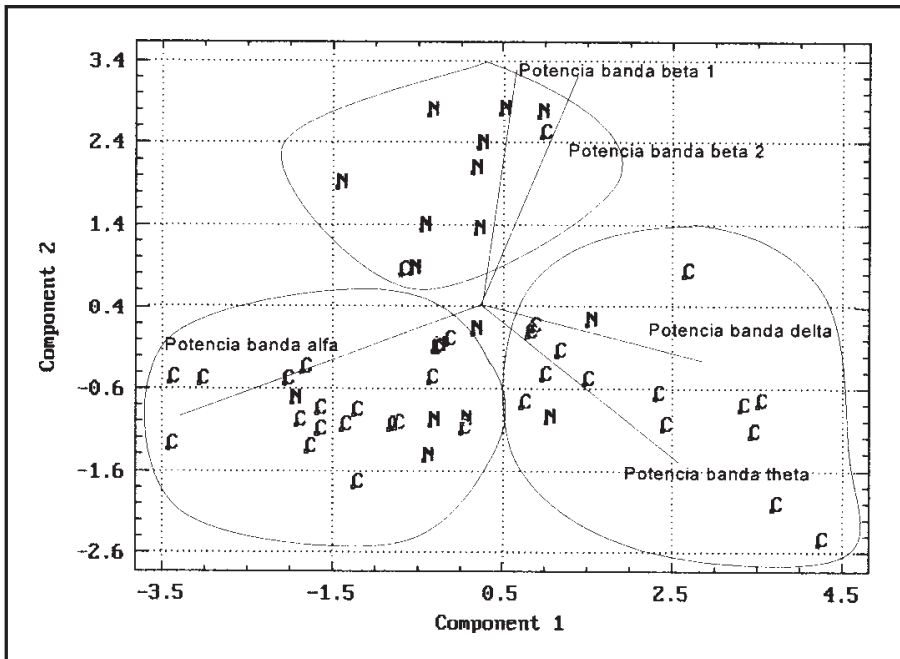

Fig 1. Análisis de componentes principales que muestra tres grupos, uno formado por los controles, en relación con el eje de la banda beta y dos conformados por los enfermos, uno de ellos en el eje de las bandas lentas y otro en el eje de la banda alfa. $C$, pacientes $N$, normales

Los dos grupos en que se subdividen los pacientes en estudio lo hacen por el peso de las potencias relativas de las bandas delta, theta y alfa y por la frecuencia dominante. Un grupo con mayor frecuencia dominante y potencia relativa alfa y otro grupo con menor potencia relativa alfa, frecuencia dominante menor y aumento de las potencias relativas delta y theta.

En los gráficos se redujo el número de sujetos normales representados, dada la gran superposición de los mismos, que dificultaba notablemente la visualización.

$\mathrm{El}$ análisis discriminante muestra diferencias significativas $(\mathrm{P}=0,00004)$ entre el grupo en estudio y los controles. Los coeficientes muestran el peso relativo de las distintas variables en dicha discriminación (Tabla 4).

Tabla 4. Resultados del análisis discriminante para la comparación entre pacientes estudiados y controles.

\begin{tabular}{ccccc}
\hline $\begin{array}{c}\text { Función Discriminante } \\
1\end{array}$ & $\begin{array}{c}\text { Eigenvalue } \\
0,5929648\end{array}$ & $\begin{array}{c}\text { Porcentaje Relativo } \\
\text { Funciones Derivadas }\end{array}$ & $\begin{array}{c}\text { Correlación Canónica } \\
0,61012\end{array}$ & \\
0 & 0,6277591 & 30,263923 & DF & Nivel de significación \\
& Coeficientes de la función discriminante & 0,00004 \\
\hline & Estandarizados & No estandarizados \\
banda delta & -1.51733 & -0.18440 \\
banda theta & -0.97829 & -0.16020 \\
banda alfa & -2.29068 & -0.14879 \\
banda beta 1 & -1.47237 & -0.34359 \\
banda beta 2 & -0.46138 & -0.07742 \\
frecuencia dominante & -0.52943 & -0.32676 \\
Constante & 18.6000 & 18.6000 \\
\hline
\end{tabular}




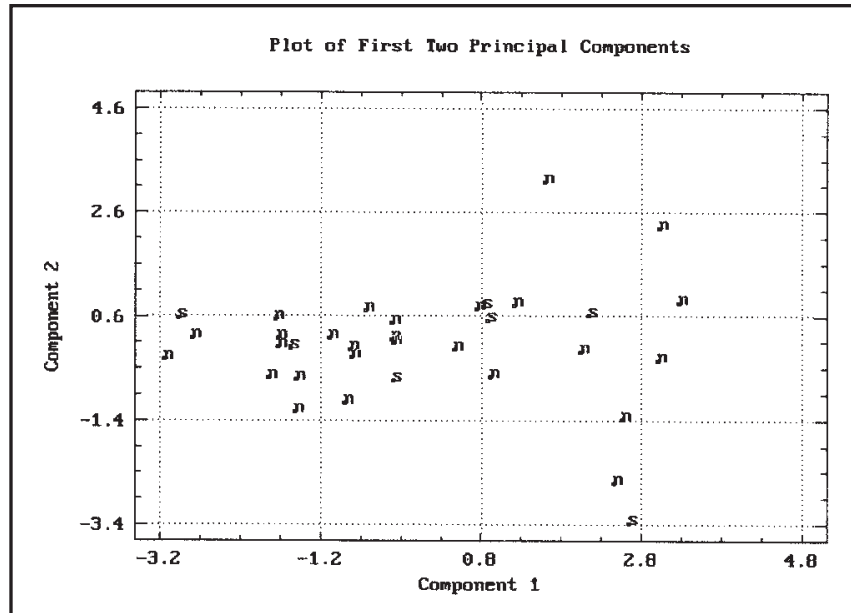

Fig 2. Análisis de componentes principales en los pacientes sin cardiopatía (n) y con cardiopatía (s) que no discrimina a ambos grupos

Se evaluaron los pacientes chagásicos, con y sin cardiopatía, por medio de un análisis de componentes principales (Fig 2) y el análisis discriminante, que confirma la ausencia de diferencias $(\mathrm{p}=0,44773)($ Tabla 5).

Proporción de estudios patológicos

Los resultados se visualizan en la Fig 3.

La edad promedio de los pacientes con potencial P 300 patológico fue de 44,7 $\pm 5,4$ años, mientras que los pacientes con potencial P 300 normal tenían una edad promedio de $43,4 \pm 8,6$ años $(\mathrm{p}=0,7627)$

Tabla 5 Resultados del análisis discriminante para la comparación entre pacientes con o sin compromiso cardíaco.

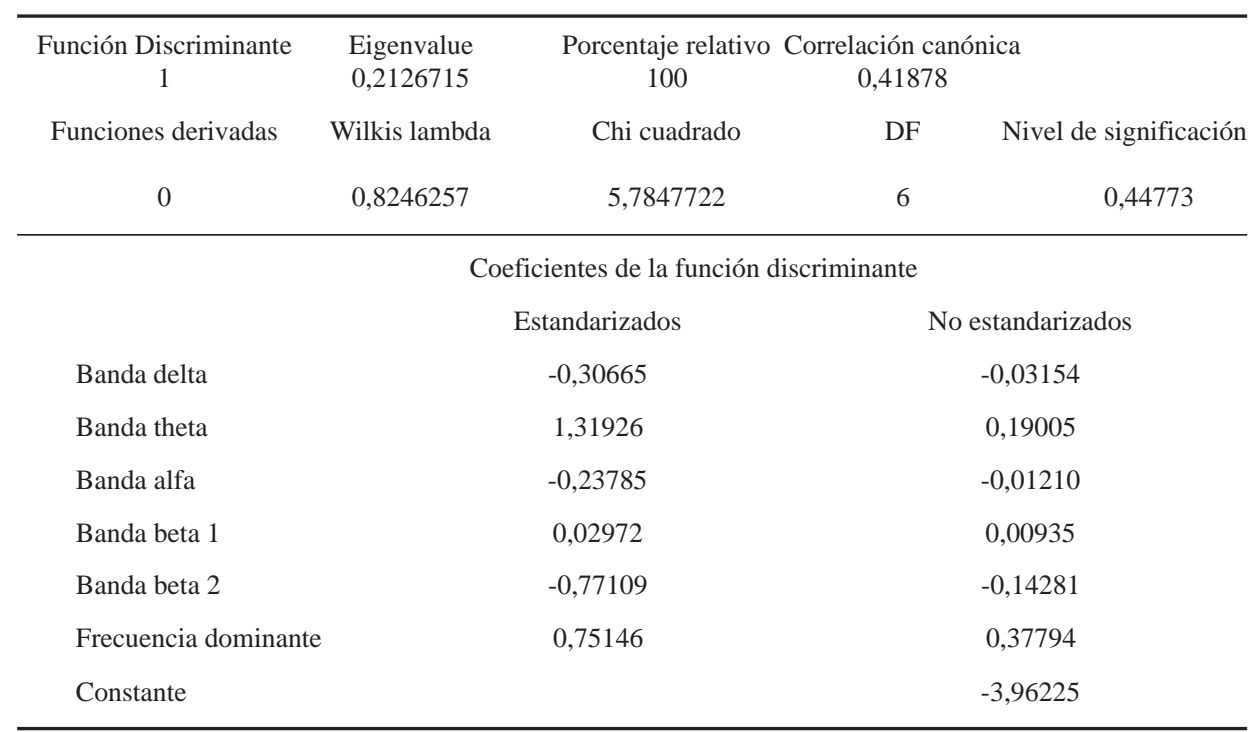




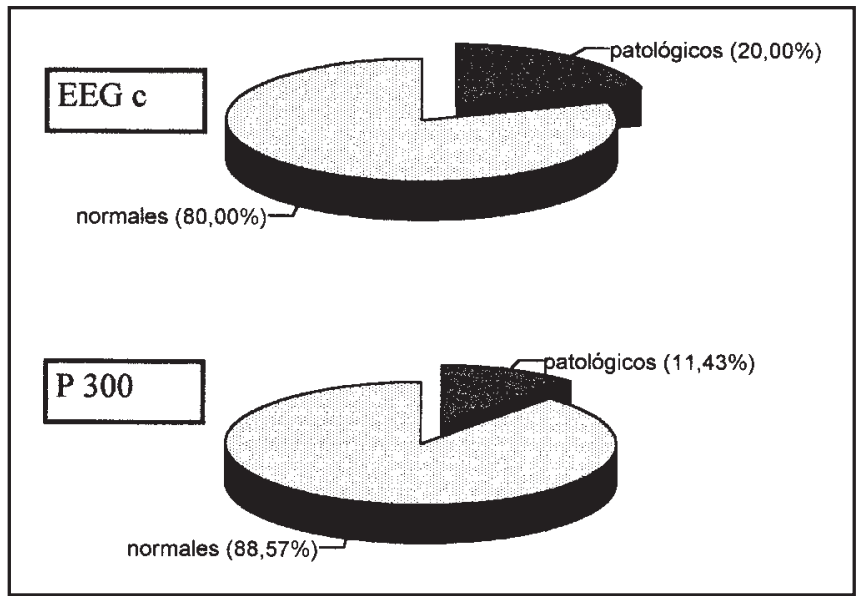

Fig 3. Proporción de resultados patológicos obtenidos por medio de los dos métodos empleados

Los EEGc patológicos se observaron en pacientes con edad media de 48,3 $\pm 5,2$ años, mientras que los pacientes con EEGc normal tenían una edad media de 42,74 $\pm 8,7$ años ( $\mathrm{p}=0,0936$ )

\section{DISCUSIÓN}

El potencial P 300 mostró latencias mayores en los pacientes que en el grupo control. A pesar de la gran superposición de los valores dicha diferencia resulta estadísticamente significativa.

La comparación entre grupos, por medio de este potencial ha sido utilizada en diversas situaciones clínicas, con similares interpretaciones respecto a la significación de este hallazgo ${ }^{23-26}$.

La amplitud de la misma onda, que no mostró diferencias significativas en este estudio, se relaciona mas con aspectos afectivos que decididamente cognitivos ${ }^{19,27}$. Cuando se consideran los resultados individualmente, se observa que el 11,43\% de los estudios muestran latencias superiores a los tres desvíos standard correspondientes para la edad. Las edades de dichos pacientes no difieren de aquellos con estudios normales.

Dada la alta especificidad del método (superior al $95 \%$ al fijar valores normales al 3 d.s.) ) $^{22,28}$ este hallazgo debe considerarse un indicador de enlentecimiento de la función cognitiva de estos pacientes.

El EEGc mostró en el análisis univariado dos diferencias que merecen ser comentadas por separado. La frecuencia dominante o frecuencia principal es menor en mas de $1 \mathrm{~Hz}$ en el grupo en estudio. Este parámetro indica cual es la frecuencia donde se concentra la mayor potencia. En condiciones normales se encuentra ubicada en la banda alfa y, como se observa en el grupo control, por encima de los $9 \mathrm{~Hz}$. La disminución de la misma es un indicador del enlentecimiento de la actividad de fondo del electroencefalograma y un importante marcador de deterioro de la actividad cerebral. Si bien ambos grupos presentan su frecuencia dominante dentro de la banda alfa, la diferencia observada, sin ser categórica, es un elemento sugestivo de cierto tipo de compromiso en los pacientes chagásicos.

Hemos observado notables diferencias en la potencia relativa de la banda beta, en especial beta 1, la cual se considera como la menos contaminada por artefactos (por ejemplo musculares). Si bien se ha emparentado habitualmente a este componente con la ansiedad, debemos recordar que también se ha 
relacionado el aumento de la potencia de la misma con el envejecimiento normal, mientras que un decremento de la misma se ha observado en relación con deterioro de las funciones cognitivas ${ }^{29}$ Esta última interpretación, si bien resulta interesante, no está totalmente aceptada, por lo cual no podemos considerarla como un indicador claro de alteración cognitiva en el grupo en estudio. Este hallazgo queda por lo tanto sin una explicación clara, la cual demandará futuras investigaciones. No se observaron focalizaciones ni asimetrías, lo cual sugiere una afectación global. Resulta más rico y categórico el análisis multivariado ${ }^{30}$, en el cual surge una franca diferencia entre los grupos.

En el análisis de componentes principales ${ }^{31}$ son claras las diferencias entre tres grupos de individuos. Los sujetos control se distribuyen en un área del plano multivariado fundamentalmente por el peso de las potencias de la banda beta y la frecuencia dominante. Los pacientes estudiados, concentrados en la dirección contraria, se dividen a su vez en dos grandes subpoblaciones, las cuales se encuentran condicionadas por la relación de ondas alfa y ondas lentas (delta y theta) cuyos vectores se oponen.

Un subgrupo tiene menor potencia relativa alfa, mayor potencia relativa delta y theta y menor frecuencia principal, características estas del EEGc en los pacientes con deterioro cognitivo. En cambio, el otro subgrupo, con incremento alfa y menor potencia relativa de ondas lentas presenta similitudes a los hallazgos habituales en cuadros depresivos ${ }^{32}$. Si los primeros correspondían al patrón que se esperaba, considerando la hipótesis de deterioro cognitivo, el hallazgo de este otro grupo no tiene una explicación a la luz de los conocimientos actuales.

$\mathrm{Al}$ analizar los resultados individuales, buscando exclusivamente aquellos que presentaran claros marcadores de deterioro bioeléctrico cerebral, comprobamos que la quinta parte de los sujetos en estudio podían incluirse en este grupo. No se observaron diferencias significativas en la edad de los pacientes con EEGc alterado, respecto a los que presentaron un estudio normal. Las diferencias entre esta proporción y la obtenida por medio del potencial P 300 es esperable por la menor sensibilidad de este último ${ }^{22,28}$. Si bien en estudios anteriores efectuados por medio de pruebas neuropsicológicas ${ }^{15} \mathrm{se}$ encontraron alteraciones en el mismo sentido, los autores plantearon sus reservas, especialmente por las influencias del nivel de instrucción de los sujetos, en los resultados de estas pruebas. El potencial P 300 puede modificarse por este parámetro, mientras que el mapeo cerebral es relativamente independiente del mismo.

Estudios recientes con tomografía computada no pudieron demostrar lesiones focales, aunque han verificado la existencia de atrofia cerebral, mayor a la esperada para la edad de los sujetos ${ }^{33}$. Si relacionamos los resultados del presente estudio, con este hallazgo neuroradiológico y con las pruebas neuropsicológicas, resulta evidente que existe una alteración cerebral en el estadío crónico de la enfermedad de Chagas y que este cursa con un deterioro de las funciones cognitivas. La proporción de individuos afectados resulta superior al número de aquellos con alteración clínica del sistema nervioso periférico $(10,17-11,24 \%)^{34,35}$, pero similar a la evidencia electrofisiológica del mismo, mayor al compromiso cordonal posterior evaluado por potencial evocado somatosensitivo $(12,5 \%)$ y menor a la troncal, observada en los potenciales evocados auditivos de tronco $(40 \%)^{14}$.

No encontramos relación entre el compromiso cerebral y miocárdico. La existencia de diferentes formas clínicas de enfermedad de Chagas, asi como la variación intraespecífica del T. cruzi (tropismo, virulencia, inmunogenecidad) son conocidas. Las distintas cepas generan una respuesta inmune diferente, y variabilidad en la relación huésped-parásito ${ }^{36-38}$. La discordancia entre compromiso nervioso y cardíaco podría ser causada por este diferente tropismo.

La presente investigación aporta una consistente evidencia sobre el compromiso cerebral en los estadios crónicos de la enfermedad de Chagas. Los mismos se inscriben en la ya numerosa serie de hallazgos de compromiso de distinta índole en el sistema nervioso central en esta afección.

Quedan numerosos interrogantes respecto a los presentes resultados, que deberán ser dilucidados en investigaciones posteriores. Deberemos tratar de establecer por ejemplo el significado de las diferencias en las bandas rápidas en el mapeo cerebral, así como definir en la medida de lo posible los factores que 
determinan la distribución de los enfermos en dos grupos, con patrones totalmente disímiles. Resulta interesante determinar igualmente si los hallazgos son de tipo secuelar o se trata de una afección evolutiva y que eventualmente pueda ser modificada por algún tipo de terapéutica.

\section{CONCLUSIONES}

Los pacientes en estadio crónico de la enfermedad de Chagas presentan diferencias electrofisiológicas en el EEGc y en el potencial P 300, que evidencian un compromiso cerebral en esta enfermedad.

En el 11,43\% de los pacientes (potencial P 300) o en el 20,00\% de los mismos (EEGc) presentan signos de dicho compromiso.

Estos hallazgos no se relaciona con la presencia de miocardiopatia.

Los mismos se suman a los precedentes (pruebas neuropsicológicas, tomografía computada) permitiendo esbozar la presencia de un sindrome cerebral en el estadio crónico de la enfermedad.

Se detectaron otras diferencias en el EEGc, como menor potencia de la banda beta o la existencia de un subgrupo de pacientes con mayor potencia relativa alfa, las cuales deberán ser explicadas en el futuro.

\section{REFERENCIAS}

1. Di Lorenzo GA, Pagano MA, Taratuto AL, Garau ML, Meli FJ, Pomsztein MD. Chagasic granulomatous encephalitis in immunosuppressed patients: computed tomography and magnetic resonance imaging findings. J Neuroimaging 1996;6:94-97.

2. Rocha A, Meneses AC, Silva AM, et al. Pathology of patients with Chagas disease and acquired inmunodeficiency syndrome. Am J Trop Med Hyg 1994;50:261-268.

3. Del Castillo M, Mendoza G, Oviedo J, Perez Bianco RP, Anselmo AE, Silva M. AIDS and Chagas' disease with central nervous system tumor-like lesión. Am J Med 1990;88(6):693-694.

4. Libaak NE, Gonzalez MI, Gutfraind E, Wainstein JM, Simone A, Carvallo O. Mielomeningoencefalitis candidiásica asociada a meningitis por tripanosoma cruzi en un paciente portador de SIDA. Rev Asoc Med Argent 1993;106:4-8.

5. Velasquez JN, Corti M, Perez Blanco R, et al. Manifestaciones neurológicas en pacientes con enfermedad de Chagas y sindrome de inmunodeficiencia adquirida. Medicina 1997;57(Supl 3):41-42.

6. Pagano M, Segura M, Di Lorenzo G, et al. Chagasic granulomatous encephalitis in acquired inmunodeficiency syndrome (AIDS). J Neurol Sci 1997;150(Suppl)S208.

7. Losavio A, Jones MC, Sanz OP, et al. A sequential study of the peripheral nervous system involvement in experimental Chagas' disease. Am J Trop Med Hyg 1989;41:539-547.

8. Mirkin GA, Jones M, Sanz OP, Rey R, Sica RE, Gonzalez Cappa SM. Experimental Chagas' disease: electrophysiology and cell composition of the neuromyopathic inflammatory lesions in mice infected with a myotropic and pantropic strain of trypanosoma cruzi. Clin Immunol Immunopathol 1994;73:69-79.

9. Jörg ME. Cefalea chagásica: sindrome mínimo de tripanosomiasis cruzi crónica. El Día Médico 1978;50:444-448.

10. Jardim E. Forma nerviosa crónica de la enfermedad de Chagas. Rev Neurol Arg 1977;3:429-438.

11. Jörg ME, Guedes Bustamante A, Peltier YA, et al. Disfunción cerebral mínima como secuela de meningoencefalitis aguda por trypanosoma cruzi. Pren Med Arg 1972;59:1658-1669.

12. Jörg ME, Orlando AS. Encefalopatía en la tripanosomiasis cruzi crónica. Pren Med Arg 1967;54:1665-1681.

13. Pereyra Käfer J, Poch G, Monteverde D, Blanco E, Tarsia R. Las manifestaciones neurológicas en la forma crónica de la enfermedad de Chagas. Rev Neurol Arg 1961;9:199.

14. Genovese O, Sanz OP, Correale J, García Erro M, Sica REP. Cerebral evoked potentials in human chronic Chagas disease. Arq Neuropsiquiatr 1989;47:274-278.

15. Pereyra SA, Mangone C, Segura E, Sica REP. Rastreo cognitivo en pacientes con enfermedad de Chagas en estadio crónico: estudio preliminar. Rev Neurol Arg 1992;17:37.

16. Mangone C, Pereyra SA, Genovese O, Segura E, Sica REP Neuropsychological impairment in chronic Chagas disease. Neurology 1992;42(Suppl 3):278.

17. Mangone C, Sica REP, Pereyra S et al. Cognitive impairment in human chronic Chagas' disease. Arq Neuropsiquiatr 1994;52:200-203.

18. Oken BS Endogenous event-related potentials. In Chiappa KH (ed). Evoked potentials in clinical medicine. New York:Raven Press, 1990:563-592.

19. Gilmore R. Evoked potentials in the elderly. J Clin Neurophysiol 1995;12:132-138

20. Polich J, Kok A. Cognitive and biological determinants of P300: an integrate review. Biol Psychol 1995;41:103-146.

21. Gasser T, Bacher P, Steinber H. Tes-retest reliability of spectral parameters of the EEG. Electroencephalogr Clin Neurophysiol 1985;60:312-319.

22. Prost JO, García AM. Diagnóstico del deterioro cognitivo por medio del potencial P 300: valoración de la sensibilidad y especificidad del método en su uso clínico. Rev Neurol Arg 1997;22:102-107. 
23. Solliway BM, Schaffer A, Pratt H, Yannai S. A multidisciplinary study of lead-exposed subjets: I. Delayed targ et detection P300 latency, an electrophysiological parameter, correlates with urinary delta-ALA. Environ Res 1994;67:168-182.

24. Quatrale R, Panarelli M, Monetti VC et al. A neurophysiological study on the P300 component of event related potentials in Hakim-Adams syndrome. Eur Neurol 1993;33:44-47.

25. Arendt G, Hefter H, Jablonowski H. Acoustically evoked event-related potentials in HIV-associated dementia. Electroencephalogr Clin Neurophysiol 1993;86:152-160.

26. Ito J, Suwazono S, Kimura J, Shibasaki H. Auditory event-related potentials in patients with systemic lupus erythematosus. Eur Neurol 1993;33:373-377.

27. Ditraglia GM, Polich J. P300 and introverted/extroverted personality types. Psychophysiology 1991;28:177-184.

28. Filipovif SR, Kostif VS. Utility of auditory P 300 in detection of presenile dementia. J Neurol Sci 1995;131:150-155.

29. Riquelme LA. Envejecimiento y demencia. In Ferrero RGA, Ferrero A (eds) Análisis computado del EEG. Buenos Aires:FADEC,1995:195-200.

30. Salgado PA, Lombardo RJ. El uso de la estadística en electrofisiología. In Ferrero RGA, Ferrero A (eds)Análisis Computado del EEG. Buenos Aires:FADEC,1995:91-110.

31. Dawson Saunders B, Trapp RG. Bioestadística médica, Mexico:El Manual Moderno,1993.

32. Riquelme LA. Aplicaciones del qEEG en la depresión. In Ferrero RGA, Ferrero A (eds) Análisis computado del EEG. Buenos Aires:FADEC,1995:201-204.

33. Lepera S, Wainstein JM, Vallasa M, et al. Silent cerebral lesions in patients with chronic Chagas disease. Preliminary data. J Neurol Sci 1997;150(Suppl):S221.

34. Sica REP. Neuromuscular involvement in chronic American trypanosomiasis. Rev Neurol Arg 1997;22(Suppl 1):81.

35. Sica REP. Compromiso del sistema nervioso. In Storino R, Milei (eds) Enfermedad de Chagas. Buenos Aires:DOYMA,1994:303-320.

36. Gonzalez Cappa S, Tekiel V, Celentano A, Mirkin G. Variación intraespecífica de trypanozoma cruzi en inmunopatología. Medicina 1997;57(Suppl 3):37-38.

37. Diego JA, Penin P, Rey J, Mayer R, Gamallo C. A comparative pathological study of three strains of Trypanosoma cruzi in an experimental model. Histol Histopathol 1991;6:199-206.

38. Postan M. Expresión de la hetrogeneidad del Trypanosoma cruzi in vivo. Medicina 1997;57(Suppl 3):38-39. 